\title{
Taking Dynamic Systems and Controls Laboratories One Step Further
}

\author{
Bill Diong*, Connie Kubo Della-Piana* and Ryan Wicker ${ }^{\dagger}$ \\ The University of Texas at El Paso
}

\begin{abstract}
The past decade has been a time of significant changes for many fields of engineering, including control systems engineering. Firstly, a paradigm shift has been occurring with regard to the kind of engineering graduates needed by today's fast-paced global economy. Secondly, the price to performance ratio of computing power has been rapidly decreasing.

As a result, when faculty in the respective Departments recently decided to incorporate laboratory experiments into the lecture-only Electrical Engineering (EE) Controls course and to update the Mechanical Engineering (ME) Controls laboratory course experiments, the opportunity arose to develop a Dynamic Systems and Controls laboratory using state-of-the-art equipment to provide a present-day-relevant education. In particular, we aimed to enhance student learning of a model-based, simulation-oriented approach to control systems analysis, design and development, and to provide them with the experience of implementing Digital Signal Processor (DSP)-based controllers.
\end{abstract}

In 1999, we received funding from the National Science Foundation's Course, Curriculum and Laboratory Improvement program to develop such a laboratory with the following expected student outcomes. First, students will become adept at using common industry software packages for system modeling, analysis, control design and simulation. Second, students will learn how to use common industry measurement instruments as well as techniques for frequency-domain modeling, analysis and control design purposes when working with hardware. Third, and most importantly, they will gain experience in using a DSP development system to implement the control algorithms they have designed for the given electromechanical systems.

The last item distinguishes this project from other recent similar projects that allow students to experiment with 'canned' DSP-based controllers but do not let them gain an understanding of, and hands-on experience with, the final implementation step in the control design and development process. Such an omission, we feel, results in a less than adequate preparation of today's student. Moreover, the cost and effort of taking students that one step further is decreasing as less expensive, more powerful, and easier to use development tools come on the market.

This paper details the objectives and accomplishments of this project and discusses preliminary findings on how this project impacts student learning for the two Controls courses.

\footnotetext{
* Department of Electrical and Computer Engineering

* Model Institutions for Excellence (Partnership for Excellence in Teacher Education)

$\dagger$ Department of Mechanical and Industrial Engineering

Proceedings of the 2001 American Society for Engineering Education Annual Conference \& Exposition Copyright (C) 2001, American Society for Engineering Education
} 


\section{Introduction}

The University of Texas at El Paso (UTEP) is a comprehensive four-year state-supported and public co-educational institution that is located in the city of El Paso, Texas. About $70 \%$ of UTEP's engineering students are Hispanic. A majority of them are also first generation college students from humble backgrounds. A significant minority work at least part-time, outside the university, to support themselves and their families. Compounding this challenging situation is a liberal admission policy that has resulted in classes with students spanning a wide range of ability and preparation. Many of them show a lack of confidence in their abilities and find it difficult to relate lecture material to real-world problems, especially in courses that tend to be more mathematically intensive. On the other hand, they appear more motivated and do better in our existing laboratory courses and in courses that use computers for solving problems, i.e., they enjoy hands-on experience and learn better that way.

We felt that re-doing the Dynamic Systems and Controls Laboratory will help greatly to stimulate the students' interest, boost their self-confidence, improve their understanding of the lecture material and prepare them better for a rewarding engineering career.

To achieve this, a proposal was recently submitted to the National Science Foundation's Course, Curriculum and Laboratory Improvement Program ${ }^{1}$ for the establishment of a state-of-theart Dynamic Systems and Controls instructional laboratory at UTEP. The proposal was funded, this laboratory was developed and it is now being used by both Electrical and Mechanical Engineering undergraduate students at UTEP. Its main goals are to:

a. prepare students to be multidisciplinary in their thinking

b. introduce students to a model-based simulation-oriented approach to control systems design and development

c. let students gain experience with the actual equipment needed for digital signal processor (DSP)-based controller implementation, and

d. allow students to demonstrate their implemented controllers on a non-trivial hardware system.

The last two objectives distinguish this project from other recent similar projects that allow students to experiment with 'canned' DSP-based controllers but do not let them gain an understanding of, and hands-on experience with, the final implementation step in the control design and development process. Such an omission, we feel, results in a less than adequate preparation of today's student. Moreover, the cost and effort of taking students that one step further is decreasing as less expensive, more powerful, and easier to use development tools come on the market.

\section{Laboratory Equipment: Hardware and Software}

The key requirement driving the laboratory setup to service the courses that will benefit from this lab is to have an open, integrated controller development platform that allows plant modeling, analysis, control design, system simulation, controller implementation and control verification. Such a platform has emerged only recently because of the tremendous gains in DSP technology and a simultaneous reduction in its cost. Furthermore, it is becoming widely adopted in industry as product development time and cost become more pressing concerns in an 
increasingly competitive global marketplace. Hence, we believe that the laboratory will not only serve as a means of education but also as a place of training to prepare our students to quickly contribute to their new employers.

The UTEP Dynamic Systems and Controls laboratory is equipped with the following: a. Matlab, Control System and Symbolic Math toolboxes, Simulink and Real-Time Workshop b. DSP development systems and boards with analog outputs suitable for driving DC motors c. dual-channel dynamic signal analyzers/function generators /oscilloscopes

d. Inverted Pendulum systems

Matlab, a product of The MathWorks Inc. ${ }^{2}$, represents the software platform underpinning the functions of almost all other equipment in the laboratory. It interfaces with a measurement and analysis module (described below) to provide visualization of the data acquired by the module. It is also used for system modeling, analysis, control design, simulation, and to provide an interface to a DSP control development system (also described below). This configuration allows one to graphically put together a model of the control algorithm, generate DSP code using that model and then download that code to the DSP. The software is available, for educational purposes, at a substantial discount. The alternatives to Matlab that were considered were ISI's MATRIX $\mathrm{X}_{\mathrm{X}}$ and Boeing's EASY5; however these are not as widely adopted or as broadly supported by thirdparties as Matlab is.

The DSP development system represents the cornerstone of all hardware in the laboratory. It is used to generate code implementing the desired control algorithms for the plant systems for download to the DSP, and to provide the actual control signals for driving those plants. When compared with similar systems, the TMS320C31 DSP-based DS1102 system from dSPACE Inc. ${ }^{3}$ was found to be the most suitable from both the cost and functionality standpoints.

The equipment in the laboratory also includes the SigLab 20-22 measurement and analysis module from the MTS Systems Corporation's DSP Technology division ${ }^{4}$ which, with the aid of Matlab, implements the following instruments with graphical user interfaces: swept-sine analyzer, oscilloscope, function generator, spectrum analyzer, network analyzer and lattice system identifier. It provides all of the basic measurement and signal generation functions required for this laboratory in a single, small, portable and inexpensive package. While other monolithic-type instruments were considered, e.g., the HP 35670A dynamic signal analyzer, they were considered too expensive to have one each per laboratory station and had substantial capabilities that would rarely be utilized in an undergraduate laboratory, and so were dropped from consideration.

Various plant systems were evaluated. The vendors included Feedback Inc., Quanser Consulting and Educational Control Products (ECP) ${ }^{5}$. The systems need to be easy, and robust enough, for the students to work with yet present fairly challenging control problems with visible results. We finally selected the ECP Inverted Pendulum system as the main plant system for this laboratory as it satisfies all the above requirements. We considered, but decided against, ordering these systems with their own proprietary control software. This is because, as previously mentioned, a major objective of this proposed lab is to have the students learn how to perform Matlab-based code development for a DSP controller as part of an integrated design and implement process. 
To run the described software and hardware in this laboratory are 10 Pentium II PCs that were donated by the Hewlett-Packard Foundation. A network server and printer, also donated by that Foundation, round out the list of equipment available in this lab.

The following figure shows the hardware configuration for each station in this laboratory.

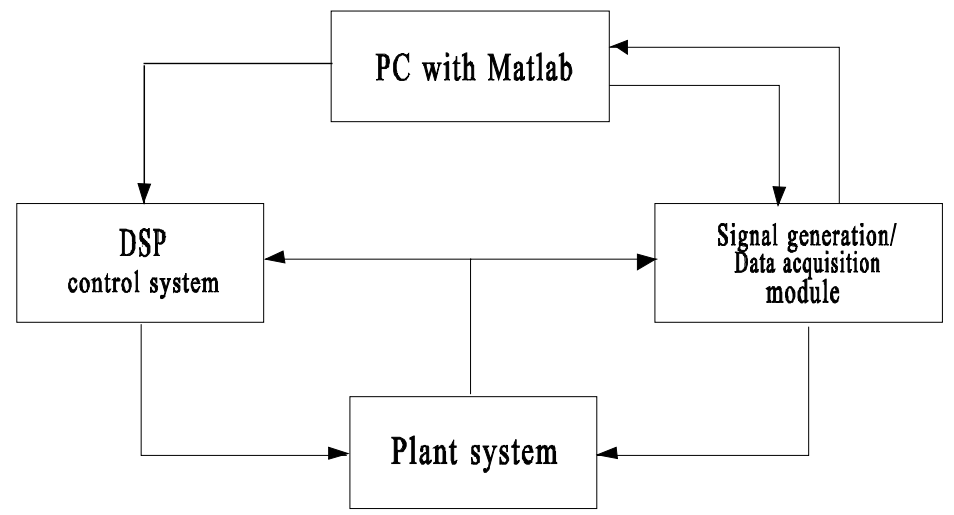

Figure 1 - Dynamic Systems and Controls laboratory station configuration

\section{Impact on UTEP's Electrical and Mechanical Engineering programs}

Our plan to motivate students in their study of engineering, to familiarize them with a model-based, simulation-oriented approach to control systems design and development, and also to provide them with experience in implementing DSP-based controllers revolves around the following proposed demonstrations and experiments. They are organized according to the course under which they will be carried out.

Demonstrations

UNIV 1301 - Seminar/Critical Inquiry : This is a required course that serves to introduce freshman students to the various scholarly disciplines. Demonstrations of various control experiments should prove to stimulate the students' interest in the study of engineering as a means of understanding and solving real-world technical problems. It also represents an opportunity to introduce the students to the idea that present-day engineers need to be multidisciplinary in their thinking.

\section{Experiments}

EE 4364 - Systems and Control : This is the elective introductory controls course for electrical engineering students. We plan to complement the existing lectures with laboratory experiences related to the following concepts:

a. frequency domain modeling

b. transient performance and steady-state performance

c. Bode plots for stability analysis and controller design

d. evaluation of various controllers for the inverted pendulum system (in default configuration) with introduction to the DSP development system and control implementation 
e. modeling, analysis, control design, simulation and DSP controller implementation for the inverted pendulum system (configured differently from above)

EE 4220 \& 4230 - Senior Project I \& II : A major goal of these courses is to develop a vehicle for the realization of the complete integrated process of system conception, design, fabrication, and operation. We intend to regularly organize year-long projects in which the student group will be faced with the conceptualization, design, fabrication, and implementation (including controller design - both hardware and software) of a system to satisfactorily perform a specified task.

MECH 4311 - Automatic Controls : The course presently relies on example problems to demonstrate the impact of control on system stability and performance. The laboratory will provide the students hands-on experience to model different mechanical, electrical, thermal and fluid systems, and to design compensators to reinforce the concepts learned in lecture. Laboratory experiments for this class will run parallel to the lecture material covered and include:

a. system modeling of components

b. modeling of integrated systems

c. time response studies

d. studies on the effect of gear ratio, inertia, friction, backlash and sampling period

e. design of compensators and performance evaluation

f. system identification via frequency response methods

g. controller design for an inverted pendulum system

MECH 4466 - Senior Design : A major goal of this course is to develop a vehicle for the realization of the complete integrated process of system conception, design, fabrication, and operation. We intend to regularly organize semester-long projects in which the student group will be faced with the conceptualization, design, fabrication, and implementation (including controller design - both hardware and software) of a system to satisfactorily perform a specified task.

\section{Progress of Laboratory Development}

The initial focus of this laboratory's development was to make it ready for demonstrations to students. As of March 2001, the following have been accomplished :

a. all of the required software and hardware has been purchased

b. the procedure for the frequency domain measurement and modeling experiment (on an RLC circuit) has been completed and a demonstration has been conducted for the Fall 2000 class of ME Automatic Controls students

c. control algorithms for the inverted pendulum, have been implemented on DSP and tested successfully

d. the procedure for the transient response experiment (PD control of the inverted pendulum) has been completed and a demonstration has been conducted for the Fall 2000 class of ME Automatic Controls students and 1 section of the Fall 2000 class of UNIV 1301 students, as well as for the public during Engineering Open House in March 2001

e. the procedure for introduction to the DSP development system and control implementation has been completed

f. a preliminary survey of both EE and ME Controls students has been performed; its results are provided and discussed below 
g. the lab is presently being used by the Spring $2001 \mathrm{EE}$ and ME Controls students for performing experiments

h. a Web site ${ }^{6}$ to disseminate information about the laboratory, with text, pictures and movies, is almost complete.

The remaining tasks that will be accomplished by the end of June 2001 include

a. completing the procedures for all of the experiments to be conducted in the Control courses

b. putting all experimental procedures on the laboratory's Web site

c. performing an evaluation of this re-developed lab course for the Spring 2001 semester

d. writing a final report on the project.

\section{Evaluation}

Our plan for evaluating the project, after the laboratory's 'maiden run' during the Spring 2001 semester, combines student self-assessment of the effect of lab experiences on level of interest in the course and the assessment of competence and confidence in understanding and applying course material in the implementation step in the control design and development process. Additionally, student feedback will be solicited via the laboratory's Web site after the completion of hands-on lab experiments to assess students' judgement as to how helpful the experiment or hands-on activity was in learning the course material. Students will be asked how they solved the problems and to describe the strategies they used to complete course assignments. Student assessment of the course will be collected during Spring 2001 and will be compared to the baseline data on course characteristics collected in Fall 1999. It is interesting to note, that when students attending the Fall 1999 class were asked for suggestions to improve the course, they requested hands-on activities and projects that more closely mirrored the "real world." Hands-on activities and "real world" problems will be implemented in Spring 2001.

Baseline data on students' self-assessment of interest in the course was collected during Spring 2000 (a summary is shown in Table 1). Little difference in student interest in the course and in participating in course activities were found when beginning of the semester levels were compared to end of the semester levels (means were obtained based on a $1-5$ scale). Levels of interest were slightly more positive at the end of the semester than at the beginning of the semester. Not all students responded to all the items on the questionnaire. No statistical testing of the difference was conducted.

Based on the logic of the new lab course design, it is hypothesized that differences in levels of interest in the Spring 2001 lab course will be greater than the differences found in the Spring 2000 course.

To better assess the outcomes of the design and implementation of the Controls Lab, a performance assessment of students' competence and confidence in the final implementation step in the control design and development process will be developed. Items will be added to the existing Controls Lab Questionnaire that will ask students to assess the level of helpfulness of class/lab activities in learning course concepts, procedures, equipment, software packages and relating course materials to "real world' problems. In addition, a focused study of the effectiveness of demonstrations and hands-on activities to increase interest in and understanding 
of engineering principles will be conducted.

\begin{tabular}{|l|c|c|c|}
\hline & $\begin{array}{l}\text { Start of } \\
\text { semester }\end{array}$ & $\begin{array}{l}\text { End of } \\
\text { semester }\end{array}$ & Difference \\
\hline Attending lectures & 3.36 & 3.63 & .27 \\
\hline Conducting research on controls with the professor & 2.46 & 3.27 & .81 \\
\hline Reading about control principles & 2.71 & 3.13 & .42 \\
\hline Learning new algorithms to identify control problems & 2.71 & 3.13 & .42 \\
\hline Applying algorithms to identify control problems & 2.57 & 3.00 & .43 \\
\hline Applying algorithms to solve control problems & 2.58 & 2.93 & .35 \\
\hline Learning new algorithms to solve control problems & 2.85 & 2.93 & .08 \\
\hline Solving problems to test my ideas & 2.69 & 2.81 & .12 \\
\hline Taking another course or lab in controls & 2.08 & 2.62 & .54 \\
\hline Attending lab & 2.78 & 2.60 & -.18 \\
\hline Searching for and reading additional material & 2.15 & 2.47 & .32 \\
\hline
\end{tabular}

Table 1 - Students' self-assessment of interest in the Controls course for Spring 2000

\section{Dissemination}

We intend to publish the final results of this project that includes a more detailed evaluation of its impact in an appropriate journal so as to more broadly disseminate the information about this laboratory's purpose, concept and implementation. Furthermore, the laboratory's Web site, which presently contains some information about the various experiments, pictures and descriptions of the different laboratory equipment and QuickTime movies of selected control experiments, will be regularly updated and continue to be made accessible to the public. In addition, we will continue to conduct demonstrations of the laboratory's capabilities during Engineering Open House and student recruitment visits to campus, as well as to show videotapes of our undergraduates performing the control experiments during off-campus student recruiting visits.

\section{Conclusions}

From the results of the preliminary survey, we can conclude that the establishment of a Dynamic Systems and Controls Laboratory at UTEP will be helpful in improving students' level of interest and student learning in both the EE and ME Controls courses. We strongly believe that it will also benefit students in other courses by stimulating their interest in the study of engineering.

\section{Acknowledgment}

This work is partially supported by the National Science Foundation through grant number DUE-9950723 under its Division of Undergraduate Education's Course, Curriculum and Laboratory Improvement program.

\section{Bibliography}

1. URL: http://www.ehr.nsf.gov/EHR/DUE/programs/ccli/; NSF Course, Curriculum, and Laboratory Improvement (CCLI) program.

Proceedings of the 2001 American Society for Engineering Education Annual Conference \& Exposition Copyright $(C$ 2001, American Society for Engineering Education 
2. URL: http://www.mathworks.com/; The MathWorks: Developers of MATLAB and Simulink for Technical Computing.

3. URL: http://www.dspaceinc.com/; dSPACE - Solutions for Control.

4. URL: http://www.dspt.com/; DSP Technology.

5. URL: http://www.ecpsystems.com/; Educational Control Products.

6. URL: http://www.ece.utep.edu/faculty/webbdiong/dsclab/index.html; University of Texas at El Paso College of Engineering Dynamic Systems and Controls Lab.

\section{BILL DIONG}

Bill Diong, Ph.D., has been an Assistant Professor in the Department of Electrical and Computer Engineering at The University of Texas at El Paso since 1999, where he is currently the Forrest and Henrietta Lewis Professor of Electrical Engineering. He received his B.S., M.S. and Ph.D. degrees from the University of Illinois (Urbana-Champaign), then gained valuable practical experience working as a Senior Research Engineer for Sundstrand Aerospace before returning to academia. His present research interests include control system applications and energy conversion systems.

\section{CONNIE KUBO DELLA-PIANA}

Connie Kubo Della-Piana, Ph.D., is the Director of Evaluation for the Model Institutions for Excellence and the Partnership for Excellence in Teacher Education at the University of Texas at El Paso. Both projects are supported by funds from the National Science Foundation. She has also been involved in the evaluation of projects funded by the Smithsonian Institution, The American Red Cross, the National Institutes of Health, NASA and the U.S. Department of Education. She is currently a participant in the National Learning Communities Project supported by the Pew Foundation.

\section{RYAN WICKER}

Ryan Wicker, Ph.D., is the John T. MacGuire Professor of Mechanical Engineering in the Mechanical and Industrial Engineering Department at The University of Texas at El Paso. He received his B.S. degree from the University of Texas at Austin and M.S. and Ph.D. degrees from Stanford University. His research areas include experimental fluid mechanics and internal combustion engines, and he has been at UTEP since completing his Ph.D. in 1994.

Proceedings of the 2001 American Society for Engineering Education Annual Conference \& Exposition Copyright (C) 2001, American Society for Engineering Education 